\title{
On the spectra of integral operators connected with Boltzmann and Schrödinger operators
}

\author{
By \\ Jun Uchiуama*
}

\section{§1. Introduction}

1. 1. In this work we shall study selfadjoint integral operators of the form

$$
(H f)(x)=V(x) \cdot f(x)+\int_{\Omega} K(x, y) f(y) d y,
$$

where $V(x) \geq 0$ and the perturbation term $K$ is, in general, an unbounded operator. Operators of this type appear rather often in applications. E. g., the so-called linearized Boltzmann operator and the Fourier transformed Schrödinger operator are of this kind. The continuous spectra of these operators have been investigated by many authors, e. g., Friedrichs [3] [4], and Faddeev [2], but it seems that the discrete part of the spectrum has not been studied systematically. Therefore we shall discuss chiefly the discrete part of the spectrum.

1. 2. Making use of Hermitian forms, Birman [1] investigated systematically operators of the form

$$
C=A+B,
$$

where $A \geq 0$, and $B$ is small relative to $A$. He applied the results to the study of spectra of differential operators (mainly of the Schrödinger type). In $\$ 2$ we shall study operators of the form (1.2), and obtain some information on the essential spectrum and the negative

Received March 27, 1967.

Revised May 16, 1967.

Communicated by S. Nakano.

* Mathematical Institute, Faculty of Science, Kyoto University. 
part of the spectrum. Our results are similar to those of Birman [1], but are stated in terms of operators rather than in terms of Hermitian forms. In $\S 3$ we shall give criteria for the selfadjointness of the operator (1.1). It will be shown that the study of the negative part of the spectrum of (1.1) reduces to that of the spectrum in $(-\infty,-1)$ of a bounded selfadjoint integral operator of the form

$$
\left(G_{0} f\right)(x)=\int_{\Omega} \frac{K(x, y)}{\sqrt{V(x)} \sqrt{V(y)}} f(y) d y .
$$

This result is particularly convenient in showing the finiteness of the discrete spectrum of (1.1). In $\$ 4$, without making use of the operator (1.3), we shall give a sufficient condition for the infiniteness of the discrete spectrum. The result entails that in the neighborhood of $x_{0}$ satisfying $V\left(x_{0}\right)=0$ the behavior of $V(x)$ and $K(x, y)$ plays an important role. The method is essentially the same as used by Z Zislin $[14]$, the author [11], etc. Finally, in $\$ 5$ we shall apply these results to the eigenvalue problems associated with the linearized Boltzmann operator, the Schrödinger operator and the operator of the so-called Friedrichs model.

It has been shown by Kuščer-Corngold [8] and Ukai [12] that there are an infinite number of discrete eigenvalues of the Boltzmann operator for the so-called mono-atomic gas model. But it seems to the author that Kuščr-Corngold's treatment is not rigorous from the mathematical point of view, and in Ukai's treatment, more strict estimates are required. Furthermore, both of them apply to this problem results from the theory of eigenvalue problems for ordinary differential equations, but we assume no such knowledge. As for Schrödinger operators, we shall give another proof of results obtained so far by Birman [1], Mizohata-Mochizuki [9], the author [11], etc.

\section{§. Perturbation of discrete and essential spectra}

2. 1. In this section, $\mathfrak{S}$ denotes an abstract Hilbert space.

Definition 2. 1. Let $A$ be a selfadjoint operator in $\mathfrak{S}$. $\lambda$ is said 
to belong to the essential spectrum $\sigma_{e}(A)$ of $A$, if there exists a sequence $\left\{u_{n}\right\}_{n=1,2,} . \subset D(A)^{1)}$ such that $\left\|u_{n}\right\|=1, u_{n}$ converges weakly to 0 , and $\lim _{n \rightarrow \infty}\left\|A u_{n}-\lambda u_{n}\right\|_{1}=0{ }^{2)}$ The complement of $\sigma_{e}(A)$ with respect to the spectrum $\sigma(A)$ of $A$ is called the discrete spectrum $\sigma_{d}(A)$ of $A$, and a point belonging to $\sigma_{d}(A)$ is said to be a discrete eigenvalue.

Then in terms of operators we shall show a result similar to Birman [1], Theorem 1.2, which he has given in terms of Hermitian forms.

Theorem 2.1. Let $A$ be a non-negative selfadjoint operator in $\mathfrak{S}_{2}{ }^{3)} B$ a symmetric operator, and $D(A)=D(B)$. Let $C=A+B$ be a selfadjoint operator with domain $D(C)=D(A)$. If the densely defined operator $(A+I)^{-1 / 2} B(A+I)^{-1 / 2}$ has a unique completely continuous extension $F_{1}$, then

(i) $C$ is a lower semi-bounded operator, and

(ii) $\sigma_{c}(C)=\sigma_{e}(A)$.

Remark 2. 1. In Theorem 2.1 the fact that $(A+I)^{-1 / 2} B(A+I)^{-1 / 2}$ has a dense domain follows from the essential selfadjointness of $(A+$ $\lambda I)\left.^{1 / 2}\right|_{D(A)}(\lambda \geq 0) .^{4)}$ In fact, since we have $\left.\left.(A+\lambda I)^{1 / 2}\right|_{D} \subset(A+\lambda I)^{1 / 2}\right|_{D(A)}$ $\subset(A+\lambda I)^{1 / 2}$, where $D=\bigcup_{n=1}^{\infty} E_{A}(n) \mathfrak{S}_{,}^{5)}$ we have only to show that $(A+$ $\lambda I)\left.^{1 / 2}\right|_{D}$ is essentially selfadjoint. Now for any $f \in D\left(A^{1 / 2}\right)$, putting $f_{n}=E_{A}(n) f \in D$, we have, as $n \rightarrow \infty,\left\|f_{n}-f\right\| \rightarrow 0$, and $\|\left.(A+\lambda I)^{1 / 2}\right|_{D} f_{n}-$ $(A+\lambda I)^{1 / 2} f\left\|^{2}=\int_{n}^{\infty}(\mu+\lambda) d\right\| E_{A}(\mu) f \|^{2} \rightarrow 0$. Thus the minimal closed extension of $\left.(A+\lambda I)^{1 / 2}\right|_{D}$ is an extension of $(A+\lambda I)^{1 / 2}$, and so $(A+$ $\lambda I)\left.^{1 / 2}\right|_{D}$ is essentially selfadjoint.

Proof. Since $F_{1} \supset(A+I)^{-1 / 2} B(A+I)^{-1 / 2}, \quad D(A) \subset D\left(A^{1 / 2}\right)$, and since $D\left((A+I)^{-1 / 2} B(A+I)^{-1 / 2}\right)=(A+I)^{1 / 2} D(A)$, we have for any $f \in$ $D(A)$

1) $D(A)$ denotes the domain of $A$.

2) Here and in the sequel, \|\| and $($,$) are used to denote the norm and inner$ product in $\mathfrak{K}$ as well as in $L^{2}(\Omega)$ introduced in $\S 3$.

3) $A$ is said to be non-negative if, for any $f \in D(A),(A f, f) \geq 0$.

4) By $\left.A\right|_{D}$ is meant the operator $A$ with its domain restricted to $D \subset D(A)$.

5) $E_{A}(\mu)$ denotes the right-continuous resolution of the identity associated with $A$. 
$(B f, f)=\left((A+I)^{1 / 2} F_{1}(A+I)^{1 / 2} f, f\right)=\left(F_{1}(A+I)^{1 / 2} f,(A+I)^{1 / 2} f\right)$.

Since $F_{1}$ is completely continuous, for any $\varepsilon>0$ there exist a finite number of elements $\left\{\varphi_{i}\right\}_{i=1 \cdots N} \subset \mathfrak{g}$ such that

$$
\begin{gathered}
\left|\left(F_{1}(A+I)^{1 / 2} f,(A+I)^{1 / 2} f\right)\right| \leq \varepsilon\left\|(A+I)^{1 / 2} f\right\|^{2} \\
+\left\|F_{1}\right\| \sum_{i=1}^{N}\left|\left((A+I)^{1 / 2} f, \varphi_{i}\right)\right|^{2} .
\end{gathered}
$$

By the density of $D(A)$ in $\mathfrak{S}$, we can choose $\left\{\tilde{\varphi}_{i}\right\}_{i=1 \cdots N} \subset D(A)$ satisfying

Thus

$$
\left\|\varphi_{i}-\tilde{\varphi}_{i}\right\| \leq \sqrt{\frac{\varepsilon}{N\left\|F_{1}\right\|}}(i=1, \cdots \cdots, N)
$$

$$
\begin{aligned}
\left|\left((A+I)^{1 / 2} f, \varphi_{i}\right)\right| & \leq\left|\left((A+I)^{1 / 2} f, \varphi_{i}-\tilde{\varphi}_{i}\right)\right|+\left|\left((A+I)^{1 / 2} f, \tilde{\varphi}_{i}\right)\right| \\
& \leq \sqrt{\frac{\varepsilon}{N\left\|F_{1}\right\|}}\left\|(A+I)^{1 / 2} f\right\|+\|f\|\left\|(A+I)^{1 / 2} \tilde{\varphi}_{i}\right\| .
\end{aligned}
$$

Consequently, for any $f \in D(C)=D(A)$, we have, with some constant $c(\varepsilon)$ depending only on $\varepsilon$,

$$
\begin{aligned}
((C+I) f, f)= & ((A+I) f, f)+(B f, f) \geq\left\|(A+I)^{1 / 2} f\right\|^{2} \\
& -3 \varepsilon\left\|(A+I)^{1 / 2} f\right\|^{2}-c(\varepsilon)\|f\|^{2} \\
= & (1-3 \varepsilon)\left\|(A+I)^{1 / 2} f\right\|^{2}-c(\varepsilon)\|f\|^{2} .
\end{aligned}
$$

If we choose $\varepsilon>0$ small enough to satisfy $0<3 \varepsilon<1$, we have assertion (i).

Choosing $\lambda>1$ large enough to satisfy $-\lambda \in \rho(A) \cap \rho(C),{ }^{6)}$ we have the resolvent equation

$$
\begin{aligned}
(A & +\lambda I)^{-1}-(C+\lambda I)^{-1} \\
& =(A+\lambda I)^{-1} B(C+\lambda I)^{-1} \\
& =(A+\lambda I)^{-1 / 2}(A+\lambda I)^{-1 / 2} B(A+\lambda I)^{-1 / 2}(A+\lambda I)^{1 / 2}(C+\lambda I)^{-1} \\
& =(A+\lambda I)^{-1 / 2} F_{\lambda}(A+\lambda I)^{1 / 2}(C+\lambda I)^{-1} .
\end{aligned}
$$

Here $F_{\lambda}$ denotes the unique completely continuous extension of $(A+$ $\lambda)^{-1 / 2} B(A+\lambda I)^{-1 / 2}$. Its existence can be seen as follows. Since $0<$ $(\mu+1)(\mu+\lambda)^{-1}<1$ for $0<\mu<\infty$, the spectral decomposition formula

6) $\rho(A)$ denotes the resolvent set of $A$. 
yields that $(A+\lambda I)^{-1 / 2}(A+I)^{1 / 2}$ has a bounded extension, which equals $(A+I)^{1 / 2}(A+\lambda I)^{-1 / 2}$, a bounded operator. Thus the complete continuity of $F_{1}$ leads to the existence of completely continuous $F_{\lambda}$ in view of

$$
\begin{aligned}
& (A+\lambda I)^{-1 / 2} B(A+\lambda I)^{-1 / 2} \\
= & (A+\lambda I)^{-1 / 2}(A+I)^{1 / 2}(A+I)^{-1 / 2} B(A+I)^{-1 / 2}(A+I)^{1 / 2}(A+\lambda I)^{-1 / 2} \\
\subset & (A+I)^{1 / 2}(A+\lambda I)^{-1 / 2} F_{1}(A+I)^{1 / 2}(A+\lambda I)^{-1 / 2}
\end{aligned}
$$

On the other hand, we can easily see that $(A+\lambda I)^{1 / 2}(C+\lambda I)^{-1}$ is a closed operator defined over all of $\mathfrak{S}$. Thus it is a bounded operator, and the right side of (2.1) is completely continuous. Then Weyl's theorem yields $\sigma_{e}\left((C+\lambda I)^{-1}\right)=\sigma_{e}\left((A+\lambda I)^{-1}\right)$. Thus assertion (ii) holds. (q. e. d.)

2.2. Definition 2.2. Let $A$ be a selfadjoint operator in $\mathfrak{W}_{\text {. The }}$ dimension $S(A: a, b)$ of the range of the projection operator $E_{A}(b-0)$ $-E_{A}(a)$ is called the total multiplicity of the spectrum of $A$ in ( $a$, b).

The next lemma is well-known. In Birman [1] it plays a fundamental role.

Lemma 2. 1. Let $A$ be a selfadjoint operator in $\mathfrak{S}$. Let $\tau(A)$ be the upper bound ( $+\infty$ permitted) of the dimensions of subspaces $M$ contained in $D(A)$ such that for any element $f \in M(f \neq 0),(A f$, $f)<0$ holds. Then we have

$$
S(A:-\infty, 0)=\tau(A) .
$$

Proof. First, let $S(A:-\infty, 0)=n<+\infty$. Then we can choose a set of elements $\left\{\varphi_{i}\right\}_{i=1, \ldots, n} \subset D(A)$ satisfying $A \varphi_{i}=\lambda_{i} \varphi_{i}, \lambda_{i}<0$, and $\left(\varphi_{i}, \varphi_{j}\right)=\delta_{i j}$. Putting $\varphi=\sum_{i=1}^{n} a_{i} \varphi_{i}\left(\sum_{i=1}^{n}\left|a_{i}\right| \neq 0\right)$, we have $(A \varphi, \varphi)=\sum_{i=1}^{n} \lambda_{i}\left|a_{i}\right|^{2}$ $<0$. Thus $S(A:-\infty, 0) \leq \tau(A)$. If we assume $\tau(A)>S(A:-\infty, 0)$, there exists some $\varphi \in D(A)$ satisfying $\|\varphi\|=1, E_{A}(0-0) \varphi=0$, and $(A \varphi$, $\varphi)<0$. This is a contradiction, and, therefore, we have $S(A:-\infty, 0)$ $=\tau(A)$.

In case $S(A:-\infty, 0)=+\infty$, we may show in a similar fashion 
that $\tau(A) \geq n$ for any $n$, so that $\tau(A)=+\infty$. (q. e. d.)

2.3. Making use of the avove lemma, we have the following result, similar to Birman [1], Lemma 1.3, where it is given in terms of Hermitian forms.

Theorem 2.2. Let $A$ be a positive selfadjoint operator in $\mathfrak{S}_{2}{ }^{7}$ $B$ a symmetric operator with domain $D(A)=D(B)$, and $C=A+B$ a selfadjoint operator with domain $D(C)=D(A)$. If $R(B) \subset R\left(A^{1 / 2}\right)$, ${ }^{8}$ and if the operator $A^{-1 / 2} B A^{-1 / 2}$ has a bounded selfadjoint extension $F_{0}$, then we have

$$
S(C:-\infty, 0)=S\left(F_{0}:-\infty,-1\right) .
$$

Corollary 2. 1 . If $F_{0}$ is a completely continuous operator, then $C$ has the following properties:

(i) $C$ is a lower semi-bounded operator.

(ii) $\sigma_{e}(C)=\sigma_{e}(A)$.

(iii) There exist at most a finite number of discrete eigenvalues of $C$ in $(-\infty, 0) .^{9)}$

Corollary 2.2. Let the conditions in Theorem 2.1 be satisfied. If $S(A+t B:-\infty, 0)$ is finite for any $t(-\infty<t<+\infty)$, then $F_{0}$ is completely continuous. ${ }^{10)}$

Proof of Theorem 2.2. First, let $S\left(F_{0}:-\infty,-1\right)=n<+\infty$. Then we can choose $\left\{\varphi_{i}\right\}_{i=1, \cdots, n} \subset \mathfrak{S}$ satisfying $F_{0} \varphi_{i}=\lambda_{i} \varphi_{i}, \quad\left(\varphi_{i}, \varphi_{j}\right)=\delta_{i j}$, $\lambda_{i}<-1(i=1, \cdots, n)$. Moreover, because $A^{1 / 2} D(A)$ is dense in $\mathfrak{L}$ (as is seen by using the spectral decomposition formula and by Remark 2.1), for any $\varepsilon>0$ we can find linearly independent $\left\{f_{i}\right\}_{i=1, \cdots, n} \subset D(A)$ so that the inequality

$$
\left\|A^{1 / 2} f_{i}-\varphi_{i}\right\| \leq \varepsilon \quad(i=1, \cdots, n)
$$

holds. Using (2.3), we have

$$
\left|\left(\left(F_{0}+I\right) A^{1 / 2} f_{i}, A^{1 / 2} f_{j}\right)-\left(\left(F_{0}+I\right) \varphi_{i}, \varphi_{j}\right)\right|
$$

7) $A$ is said to be positive if, for any $f \in D(A)(f \neq 0),(A f, f)>0$.

8) $R(A)$ denotes the range of $A$.

9) This corollary is similar to Birman [1], Theorem 1.4.

10) This corollary is also similar to Birman [1], Theorem 1.4. 
On the spectra of integral operators connected with Boltzmann 107

$$
\begin{aligned}
& \leq\left|\left(\left(F_{0}+I\right)\left(A^{1 / 2} f_{i}-\varphi_{i}\right), \varphi_{j}\right)\right|+\left|\left(\left(F_{0}+I\right) A^{1 / 2} f_{i},\left(A^{1 / 2} f_{,}-\varphi_{j}\right)\right)\right| \\
& \leq\left(\left\|F_{0}\right\|+1\right) \varepsilon+\left(\left\|F_{0}\right\|+1\right)(1+\varepsilon) \varepsilon=\left(\left\|F_{0}\right\|+1\right)(2+\varepsilon) \varepsilon .
\end{aligned}
$$

Since $D\left(A^{-1 / 2} B A^{-1 / 2}\right)=A^{1 / 2} D(A), B g=A^{1 / 2} F_{0} A^{1 / 2} g$ for any $g \in D(A) \subset$ $D\left(A^{1 / 2}\right)$. If we put $f=\sum_{i=1}^{n} a_{i} f_{i}, \sum_{i=1}^{n}\left|a_{i}\right| \neq 0$, then $f \in D(A)$, and we have by (2.4),

$$
\begin{aligned}
(C f, f) & =(A f, f)+(B f, f) \\
& =\left(A^{1 / 2} f, A^{1 / 2} f\right)+\left(F_{0} A^{1 / 2} f, A^{1 / 2} f\right) \\
& =\left(\left(F_{0}+I\right) A^{1 / 2} f, A^{1 / 2} f\right) \\
& =\sum_{i=1}^{n} \sum_{j=1}^{n} a_{i} \bar{a}_{j}\left(\left(F_{0}+I\right) A^{1 / 2} f_{i}, A^{1 / 2} f_{j}\right) \\
& \leq \sum_{i=1}^{n}\left|a_{i}\right|^{2}\left\{\left(\left(F_{0}+I\right) \varphi_{i}, \varphi_{i}\right)+\left(\left\|F_{0}\right\|+1\right)(2+\varepsilon) \varepsilon\right\}+ \\
& +\sqrt{n \sum_{i=1}^{n}\left|a_{i}\right|^{2}} \cdot \sqrt{n \sum_{j=1}^{n}\left|a_{j}\right|^{2}}\left(\left\|F_{0}\right\|+1\right)(2+\varepsilon) \varepsilon \\
& =\sum_{i=1}^{n}\left|a_{i}\right|^{2}\left\{\lambda_{i}+1+(n+1)\left(\left\|F_{0}\right\|+1\right)(2+\varepsilon) \varepsilon\right\} .
\end{aligned}
$$

Choosing $\varepsilon>0$ small enough, we have $(C f, f)<0$, since $\lambda_{\imath}<-1$. This shows, in view of Lemma 2.1 , that $S(C:-\infty, 0) \geq S\left(F_{0}:-\infty,-1\right)$.

Now, if we assume $S(C:-\infty, 0)>S\left(F_{0}:-\infty,-1\right)$, by Lemma 2.1 there exists a set of elements $\left\{f_{i}\right\}_{i=1, \cdots, n+1}$ such that $\left\{f_{i}\right\}_{i=1, \cdots, n+1}$ $\subset D(A),\left(f_{i}, f_{j}\right)=\delta_{i j}$, and $\left.\left(C\left(\sum_{i=1}^{n+1} a_{i} f_{i}\right)\right),\left(\sum_{i=1}^{n+1} a_{i} f_{i}\right)\right)<0$ for any $\left\{a_{i}\right\}_{i=1, \cdots, n+1}$ satisfying $\sum_{i=1}^{n+1}\left|a_{i}\right| \neq 0$. We decompose $A^{1 / 2} f_{i}$ as follows: $A^{1 / 2} f_{i}=g_{i}^{(1)}+$ $g_{i}^{(2)}$, where $g_{\imath}^{(1)} \in E_{F_{0}}(-1-0) \mathfrak{W}$ and $g_{\imath}^{(2)} \in\left(I-E_{F_{0}}(-1-0)\right) \mathfrak{W}$. Taking account of $S\left(F_{0}:-\infty,-1\right)=n$, we can choose $\left\{a_{i}\right\}_{i=1, \cdots, n+1}$ satisfying $\sum_{i=1}^{n+1} a_{i} g_{i}^{(1)}=0$ and $\sum_{i=1}^{n+1}\left|a_{i}\right|=1$. Then

$$
\begin{aligned}
0> & \left(C\left(\sum_{i=1}^{n+1} a_{i} f_{i}\right), \quad\left(\sum_{i=1}^{n+1} a_{i} f_{i}\right)\right)=\left\|A^{1 / 2}\left(\sum_{i=1}^{n+1} a_{i} f_{i}\right)\right\|^{2} \\
& +\int_{-1-0}^{\left\|F_{0}\right\|} \lambda d\left\|E_{F_{0}}(\lambda) A^{1 / 2}\left(\sum_{i=1}^{n+1} a_{i} f_{i}\right)\right\|^{2} \\
& \geq\left\|A^{1 / 2}\left(\sum_{i=1}^{n+1} a_{i} f_{i}\right)\right\|^{2}-\left\|A^{1 / 2}\left(\sum_{i=1}^{n+1} a_{i} f_{i}\right)\right\|^{2}=0
\end{aligned}
$$

is a contradiction. Thus $S(C:-\infty, 0)=S\left(F_{0}:-\infty,-1\right)$. 
In case $S\left(F_{0}:-\infty,-1\right)=+\infty$, for any $n>0$ we can similarly choose an $n$-dimensional subspace $M$ such that for any element $f \in M$, $f \neq 0,(C f, f)<0$ holds. This shows that $S(C:-\infty, 0) \geq n$. Then, by Lemma $2.1, S(C:-\infty, 0)=+\infty$. (q. e. d.)

Proof of Corollary 2. 1. By the same method that was applied in the proof of Theorem 2.1 to $F_{\lambda}$ and $F_{1}$, we can utilize the complete continuity of $F_{0}$ to show that $F_{1}$, which is the unique bounded extension of $(A+I)^{-1 / 2} B(A+I)^{-1 / 2}$, is completely continuous. Then, taking account of Theorem 2.1 and Remark 2.1, we have (i) and (ii). The assertion (iii) is clear from Theorem 2.2. (q. e. d.)

Proof of Corollary 2.2. By Theorem 2.2, $t F_{0}$ has at most a finite number of discrete eigenvalues in $(-\infty,-1)$, so that the spectrum of $F_{0}$ in $(-\infty, 0) \cup(0, \infty)$ is discrete. Thus $F_{0}$ is completely continuous. (q. e. d.)

Remark 2.2. In case that the inequality $\|B f\| \leq a\|A f\|+b\|f\|$ $(a, b>0)$ holds for any $f \in D(A)$, we may replace in Theorem 2.2 the condition $R(B) \subset R\left(A^{1 / 2}\right)$ by the condition that $A \mid A^{-1 / 2} D$ is essentially selfadjoint, where $D=D\left(A^{-1 / 2} B A^{-1 / 2}\right)$. In fact, since $A^{1 / 2} \mid A^{-1 / 2} D$ is essentially selfadjoint, and is a positive operator, $D$ is dense in $\mathfrak{S}$. Thus, examing the beginning part of the proof of Theorem 2.2, we can choose $\left\{f_{i}\right\}_{i=1, \cdots, n} \subset A^{-1 / 2} D$ satisfying (2.3), so that we have $S(C:-\infty$, $0) \geq S\left(F_{0}:-\infty, 0\right)$ in a similar fashion. On the other hand, we have $B f=A^{1 / 2} F_{0} A^{1 / 2} f$ for any $f \in D(A)$. In fact, since $A \mid A^{-1 / 2} D$ is essentially selfadjoint, there exists $\left\{f_{n}\right\}_{n=1,2, \ldots} \subset A^{-1 / 2} D$ such that $\left\|f-f_{n}\right\| \rightarrow 0$ and $\left\|A f_{n}-A f\right\| \rightarrow 0$. Then $\left\|B f-B f_{n}\right\| \leq a\left\|A f_{n}-A f\right\|+b\left\|f-f_{n}\right\| \rightarrow 0$ and $\left\|A^{1 / 2} f_{n}-A^{1 / 2} f\right\|^{2}=\left(A\left(f-f_{n}\right),\left(f-f_{n}\right)\right) \rightarrow 0$. Therefore, $B f_{n}=$ $A^{1 / 2} F_{0} A^{1 / 2} f_{n}, F_{0}$ is bounded, and $A^{1 / 2}$ is closed, and hence $B f=A^{1 / 2} F_{0} A^{1 / 2} f$. Thus we can also show, in a way similar to the proof of Theorem 2. 2, that $S(C:-\infty, 0)>S\left(F_{\mathrm{c}}:-\infty,-1\right)$ is a contradiction. 


\section{§3. Perturbation of multiplicative operators by integral operators}

3. 1. Let $\Omega$ be a (not-necessarily bounded) connected domain in the $m$-dimensional Euclidean space $R^{m}$. In $L^{2}(\Omega)$ let us consider the operator

$$
\begin{aligned}
(H f)(x) & =V(x) \cdot f(x)+\int_{\Omega} K(x, y) f(y) d y \\
& =(V f)(x)+(K f)(x)
\end{aligned}
$$

On this operator, here in the sequel, we impose the following conditions.

Assumption 1. $V(x)$ is a real-valued and continuous function on $\Omega$. Let the domain of the multiplicative operator $V$ be $D(V)=$ $\left\{f \in L^{2}(\Omega): V(x) \cdot f(x) \in L^{2}(\Omega)\right\}$.

Assumption 2. $\inf _{x \in \Omega} V(x)=0$.

Assumption 3. The integral operator $K$ is generated by $K(x, y)$ $=\overline{K(y, x)}$, and is symmetric on $D(K)=D(V) \cdot{ }^{11}$

If we set $D(H)=D(V), H$ is a well-defined linear operator acting in $L^{2}(\Omega)$. In the following $H$ denotes the operator thus defined.

3. 2. Lemma 3. 1. The operator $V$ is selfadjoint, and

$$
\sigma_{e}(V)=\left[0, \sup _{x \in \Omega} V(x)\right] \text {. }^{12}
$$

Proof. By Assumption 1 we can easily see that $V$ is a selfadjoint operator in $L^{2}(\Omega)$. The right-continuous resolution of the identity $\left\{E_{V}(\lambda)\right\}$ for $V$ is given as follows: For any $\varphi(x) \in L^{2}(\Omega)$

$$
E_{V}(\lambda) \varphi(x)=\left\{\begin{array}{ccc}
0, & \text { if } & V(x)>\lambda \\
\varphi(x), & \text { if } & V(x) \leq \lambda
\end{array}\right.
$$

Then the fact that $V(x)$ is continuous in $\Omega$ and satisfies Assumption 2 leads to (3.2). (q. e. d.)

11) It seems that the condition $K(x, y)=\overline{K(y, x)}$ is independent of the symmetricity of $K$ on $D(K)=D(V)$.

12) Here the case $\sup _{x \in Q} V(x)=\infty$ is also permitted. 
Next, we prove a result useful in dealing with operators of the form (3.1).

Theorem 3. 1. Suppose there exist a real number $\alpha$ such that

$$
\int_{\Omega} \int_{\Omega} \frac{|K(x, y)|^{2}}{(V(x)+1)^{\alpha}} d x d y<+\infty
$$

holds, and for any $a>0$ there is some number $c(a)$ satisfying

$$
|V(x)|^{\alpha} \leq a^{2}|V(x)|^{2}+c(a)^{2} .
$$

Then

(i) $H$ is a lower semi-bounded selfadjoint operator in $L^{2}(\Omega)$,

(ii) $\sigma_{e}(H)=\sigma_{e}(V)$.

Proof. By (3.5) we can assume that the inequality $|V(x)+1|^{\alpha}$ $\leq a^{2}|V(x)|^{2}+\tilde{c}(a)^{2}$ with some constant $\tilde{c}(a)$ holds. For any $f \in D(V)$, we have

$$
\begin{aligned}
\|K f\|^{2} & =\int_{\Omega} d x\left|\int_{\Omega} K(x, y) f(y) d y\right|^{2} \\
& \leq \int_{\Omega} d x\left(\int_{\Omega} \frac{|K(x, y)|^{2}}{(V(y)+1)^{\alpha}} d y\right)\left(\int_{\Omega}(V(y)+1)^{\alpha}|f(y)|^{2} d y\right) \\
& \leq \int_{\Omega}\left(a^{2}|V(y)|^{2}|f(y)|^{2}+\tilde{c}(a)^{2}|f(y)|^{2}\right) d y \\
& \times \int_{\Omega} \int_{\Omega} \frac{|K(x, y)|^{2}}{(V(y)+1)^{\alpha}} d x d y \leq(a\|V f\|+\tilde{c}(a)\|f\|)^{2} \\
& \times \int_{\Omega} \int_{\Omega} \frac{|K(x, y)|^{2}}{(V(y)+1)^{\alpha}} d x d y .
\end{aligned}
$$

If we choose $a>0$ small enough to satisfy $0 \leq a^{2} \int_{\Omega} \int_{\Omega} \frac{|K(x, y)|^{2}}{(V(y)+1)^{\alpha}} d x d y$ $=\varepsilon^{2}<1$, there exists some $\tilde{\tilde{c}}(\varepsilon)>0$ such that for any $f \in D(V)$,

$$
\|K f\| \leq \varepsilon\|V f\|+\tilde{\tilde{c}}(\varepsilon)\|f\| \text {. }
$$

With the help of a lemma of Kato [7], one can show, by Lemma 3. 1, that $H$ is selfadjoint.

On the other hand, we can assume that $c(a)$ is non-increasing for $a>0$. Then there exists $a>0$ large enough to satisfy $\frac{c(a)}{a}<1$, and we have by Assumptions 2 and 3 


$$
\begin{aligned}
\int_{\Omega} \int_{\Omega} \frac{|K(x, y)|^{2}}{(V(x)+1)(V(y)+1)} d x d y \\
\quad=2 \iint_{V(x)>V(y)} \frac{|K(x, y)|^{2}}{(V(x)+1)(V(y)+1)} d x d y \\
\quad+\iint_{V(x)=V(y)} \frac{\mid K(x)+1)(V(y)+1)}{(V(x)+1)(V(y)} d x d y \leq 3 \iint_{\Omega} \frac{|K(x, y)|^{2}}{(V(y)+1)^{2}} d x d y \\
\quad \leq 3 \iint_{\Omega} \frac{|K(x, y)|^{2}}{|V(y)|^{2}+1} d x d y \leq 3 a^{2} \iint_{\Omega \Omega} \frac{|K(x, y)|^{2}}{a^{2}|V(y)|^{2}+\bar{c}(a)^{2}} d x d y \\
\quad \leq 3 a^{2} \iint_{\Omega} \frac{|K(x, y)|^{2}}{(V(y)+1)^{\alpha}} d x d y<+\infty .
\end{aligned}
$$

Thus $(V+I)^{-1 / 2} K(V+I)^{-1 / 2}$ has a completely continuous extension. Therefore, by Theorem 2.1, we have (ii). (q. e. d.)

As another direct application of Theorem 2.2 we state the following.

Theorem 3.2. Let the set $\{x \in \Omega: V(x)=0\}$ has Lebesgue measure 0 , and the integral operator

$$
\left(G_{0} f\right)(x)=\int_{\Omega} G_{0}(x, y) f(y) d y=\int_{\Omega} \frac{K(x, y)}{\sqrt{V(x)} \sqrt{V(y)}} f(y) d y
$$

be bounded, selfadjoint in $L^{2}(\Omega)$. If $H=V+K$ with $D(H)=D(V)$ is selfadjoint, then $S(H:-\infty, 0)=S\left(G_{0}:-\infty,-1\right)$.

Proof. We have only to check the conditions of Theorem 2.2: $V$ is positive, because $\{x \in \Omega: V(x)=0\}$ has measure 0 ; for $f \in$ $V^{1 / 2} D(V)$ we have $V^{1 / 2} G_{0} f=K V^{-1 / 2} f$, which implies that $R(K) \subset$ $R\left(V^{1 / 2}\right)$; the other conditions are obvious. (q. e. d.)

\section{§. On the infiniteness of the discrete eigenvalues}

4. 1. The following fact is useful in showing the infiniteness of the negative part of the spectrum.

Lemma 4.1. Let $\omega$ be a subdomain of $\Omega$. Let $H_{\omega}$ be the operator formally defined by 


$$
\begin{aligned}
\left(H_{\omega} f\right)(x) & =V(x) \cdot f(x)+\int_{\omega} K(x, y) f(y) d y \\
& =\left(V_{\omega} f\right)(x)+\left(K_{\omega} f\right)(x) \quad(x \in \omega) .
\end{aligned}
$$

Then for any $f, g \in D\left(V_{\omega}\right)=\left\{f(x) \in L^{2}(\omega) ; V(x) f(x) \in L^{2}(\omega)\right\}, K_{\omega} f \in$ $L^{2}(\omega),\left(K_{\omega} f, g\right)_{\omega}=\left(f, K_{\omega} g\right)_{\omega}$, and $H_{\omega} f \in L^{2}(\omega) .^{13)}$

Moreover, if $H$ and $H_{\omega}$ are selfadjoint operators with $D(H)=$ $D(V) \subset L^{2}(\Omega)$ and $D\left(H_{\omega}\right)=D\left(V_{\omega}\right) \subset L^{2}(\omega)$, respectively, then

$$
S\left(H_{\omega}:-\infty, 0\right) \leq S(H:-\infty, 0) .
$$

Proof. We define $\tilde{f}(x) \in L^{2}(\Omega)$ by

$$
\tilde{f}(x)=\left\{\begin{array}{c}
f(x): x \in \omega \\
0: x \in \Omega \ominus \omega
\end{array}\right.
$$

for $f \in D\left(V_{\omega}\right)$, then $\tilde{f} \in D(V)=D(K)$. Thus $K \tilde{f} \in L^{2}(\Omega)$ yields $K_{\omega} f$ $\in L^{2}(\omega)$, and $\left(K_{\omega} f, g\right)_{\omega}=(K \tilde{f}, \tilde{g})=(\tilde{f}, K \tilde{g})=\left(f, K_{\omega} g\right)_{\omega}$ by Assumption 3 .

We prove (4.2). First let $S\left(H_{\omega}:-\infty, 0\right)=n<+\infty$. By Lemma 2.1, we can choose an $n$-dimensional subspace $M \subset D\left(H_{\omega}\right)$ such that for any $f \in M(f \neq 0),\left(H_{\omega} f, f\right)_{\omega}<0$ holds. With $\tilde{f} \in L^{2}(\Omega)$ defined as above, the subspace $\widetilde{M} \subset D(H)$ spanned by $\tilde{f}$ for $f \in M$ is an $n$-dimensional subspace of $L^{2}(\Omega)$, and $(H \tilde{f, \tilde{f}})=\left(H_{\omega} f, f\right)_{\omega}<0$ for any $\tilde{f} \in$ $\tilde{M}(\tilde{f} \neq 0)$. By Lemma $2.1 S(H:-\infty, 0) \geq n$. In case $n=\infty$, we obtain $S(H:-\infty, 0) \geq n$ for any $n$ in the same way. (q. e. d.)

Remark 4.1. If $\omega \subset \Omega$ is a bounded domain and $V(x)$ is bounded on $\omega$, then with $D\left(H_{\omega}\right)=L^{2}(\omega), H_{\omega}$ is a bounded selfadjoint operator in $L^{2}(\omega)$. In fact, $V_{\omega}$ is a bounded selfadjoint operator in $L^{2}(\omega)$, and by Lemma $4.1 K_{\omega}$ is a bounded selfadjoint operator.

Remark 4.2. If for some $\varepsilon(0<\varepsilon<1)$ and some constant $c(\varepsilon)$ depending only on $\varepsilon\|K g\| \leq \varepsilon\|V g\|+c(\varepsilon)\|g\|$ holds for any $g \in D(V)$, then $H$ is a selfadjoint operator in $L^{2}(\Omega)$, and $H_{\omega}$ is a selfadjoint operator in $L^{2}(\omega)$. In fact, for any $f \in D\left(V_{\omega}\right)$ we have $\left\|K_{\omega} f\right\|_{\omega} \leq\|K \tilde{f}\|$

13) Here and in the sequel, we write $(f, g)_{\omega}=\int_{\omega} f(x) \bar{g}(x) d x$ and $\|f\|_{\omega}=(f, f)_{\omega}^{1 / 2}$ for $f, g \in L^{2}(\omega)$. 
$\leq \varepsilon_{\|}\|V \tilde{f}\|+c(\varepsilon)\|\tilde{f}\|=\varepsilon\left\|V_{\omega} f\right\|_{\omega}+c(\varepsilon)\|f\|_{\omega}$, from which, by a Lemma in Kato [7], it follows that $H_{\omega}$ is a selfadjoint operator in $L^{2}(\omega)$ with domain $D\left(H_{\omega}\right)=D\left(V_{\omega}\right)$.

4. 2. Here is a sufficient condition for the infiniteness of the discrete eigenvalues of $H$ in $(-\infty, 0)$.

Theorem 4. 1. Let $H$ be a selfadjoint operator in $L^{2}(\Omega)$. Assume that for some point $x_{0} \in \bar{\Omega}$ and some domain $\omega=\{x: x$ belongs to a cone whose vertex is $x_{0}$ and $\left.\left|x-x_{0}\right|<r_{0}\right\} \subset \Omega$, there exists real numbers $\nu>0, \quad \beta_{i}, \quad \gamma_{i}, \quad \delta_{i}(i=1, \cdots, N), \quad \beta_{i}^{\prime}, \gamma_{i}^{\prime}, \quad \delta_{i}^{\prime}\left(i=1, \cdots, N^{\prime}\right), \quad c \geq 0$, $d_{i}(i=1, \cdots, N), \varepsilon^{\prime}>0$, and functions $M(x, y)$, and $N(x, y)$ with $M(x$, $y)=\overline{M(y, x)}, N(x, y)=\overline{N(y, x)}$, such that:

$$
\begin{aligned}
& 0 \leq V(x) \leq c\left|x-x_{0}\right|^{\nu}(x \in \omega) ; \\
& K(x, y)=M(x, y)+N(x, y) ;
\end{aligned}
$$

where $\operatorname{Re}(M(x, y)) \leq-\sum_{i=1}^{N} d_{i}|x-y|^{\beta_{i}}\left|x-x_{0}\right|^{\gamma_{i}}\left|y-x_{0}\right|^{\delta_{i}}$, for $\left|x-x_{0}\right| \leq$ $\left|y-x_{0}\right|$ and $x \in \omega, y \in \omega$,

$$
\operatorname{Re}(N(x, y)) \leq \mathrm{const} \sum_{i=1}^{N^{\prime}}|x-y|^{\beta_{i}{ }^{\prime}}\left|x-x_{0}\right|^{\gamma_{i}{ }^{\prime}}\left|y-x_{0}\right|^{\delta_{t^{\prime}}} \text {, for }\left|x-x_{0}\right|
$$

$\leq\left|y-x_{\mathrm{c}}\right|$ and $x \in \omega, y \in \omega$;

$$
\begin{gathered}
\beta_{i}+\gamma_{i}+\delta_{i}=\nu-m \quad(i=1, \cdots, N) \quad \text { and } \\
\min _{1 \leq i \leq N^{\prime}}\left(\beta_{i}^{\prime}+\gamma_{i}^{\prime}+\delta_{i}^{\prime}\right)=\nu-m+\varepsilon^{\prime} ; \\
\inf _{g \in W(\omega)}\left\{c \int_{\omega}|x|^{\nu}|g(x)|^{2} d x\right. \\
\left.-2 \sum_{i=1}^{N} d_{i} \int_{|\omega| \leq|y|} \int_{\omega}|x-y|^{\beta_{i}}|x|^{\gamma_{i}}|y|^{\delta_{i}} g(x) g(y) d x d y\right\}<0,
\end{gathered}
$$

where $\widetilde{\omega}=\left\{x: x=x^{\prime}-x_{0}, x^{\prime} \in \omega\right\} \quad$ and $W(\widetilde{\omega})=\left\{g(x) \in L^{2}(\widetilde{\omega}):\|g\|_{\widetilde{\omega}}=1\right.$, $g(x) \geq 0$ for a. e. $x \in \widetilde{\omega}\}$; and

$$
\min _{\mu \in \sigma_{e}\left(H_{\omega}\right)} \mu=\min _{\lambda \in \sigma_{e}(H)} \lambda=0 .
$$

Then there exists at least one negative discrete eigenvalue of $H$.

Moreover, if we assume that

$$
K_{\omega}^{(2)}(x, y)=\int_{\omega}|K(x, z) K(z, y)| d z
$$


is a kernel of an integral operator which is bounded in $L^{2}(\omega)$, and that there exist real numbers $\beta_{i}^{\prime \prime}, \gamma_{i}^{\prime \prime}, \delta_{i}^{\prime \prime}\left(i=1, \cdots, N^{\prime \prime}\right)$, and $\varepsilon^{\prime \prime}>0$ such that

$$
K_{\omega}^{(2)}(x, y) \leqq \text { const } \sum_{i=1}^{N \prime \prime}|x-y|^{\beta_{i}^{\prime \prime}}\left|x-x_{0}\right|^{\gamma_{i}^{\prime \prime}}\left|y-x_{0}\right|^{\delta_{i}^{\prime \prime}}
$$

for $\left|x-x_{0}\right| \leq\left|y-x_{0}\right|$ and $x \in \omega, y \in \omega$, where

$$
\min _{1 \leq i \leq N^{\prime \prime}}\left(\beta_{i}^{\prime \prime}+\gamma_{i}^{\prime \prime}+\delta_{i}^{\prime \prime}\right)=\nu-m+\varepsilon^{\prime \prime},
$$

then there exist an infinite number of negative discrete eigenvalues of $H$.

4. 3. Proof. First let the conditions (4.3)-(4.7) be satisfied. According to Remark $4.1, H_{\omega}$ is a bounded selfadjoint operator in $L^{2}(\omega)$. By (4.6) we can choose some function $\tilde{g}_{1}(x)$ such that

$$
\begin{aligned}
& c \int_{\tilde{\omega}}|x|^{\nu}\left|\tilde{g}_{1}(x)\right|^{2} d x \\
& -2 \sum_{i=1}^{N} d_{i} \int_{|x| \leq|y|} \int_{\omega}|x-y|^{\beta_{i}}|x|^{\gamma_{i}}|y|^{\delta_{i}} \tilde{g}_{1}(x) \tilde{g}_{1}(y) d x d y \equiv-k_{0}<0,
\end{aligned}
$$

and

$$
\begin{aligned}
& \text { (4.12) } \int_{\widetilde{\omega}}\left|\tilde{g}_{1}(x)\right|^{2} d x=1, \tilde{g}_{1}(x) \geq 0 \text { for a. e. } x \in \widetilde{\omega} \text {, and } \tilde{g}_{1}(x) \equiv 0 \text { for } \\
& \text { Setting } \\
& \quad x \notin \widetilde{\omega} \text {. }
\end{aligned}
$$

(4.13) $\quad \tilde{g}_{l}(x)=l^{m / 2} \tilde{g}_{1}(l x)(l \geq 1)$ and $g_{l}(x)=\tilde{g}_{l}\left(x-x_{0}\right)$,

we have

(4.14) $\int_{\omega}\left|g_{l}(x)\right|^{2} d x=1, g_{l}(x) \geq 0$ for a. e. $x \in \omega$, and $g_{l}(x) \equiv 0$ for Then $x \notin \omega$.

$$
\left(V_{\omega} g_{l}, g_{l}\right)_{\omega} \leq c \int_{\dot{\omega}}|x|^{\nu}\left|\tilde{g}_{l}(x)\right|^{2} d x=c l^{-\nu} \int_{\tilde{\omega}}|x|^{\nu}\left|\widetilde{g}_{1}(x)\right|^{2} d x
$$

and by (4.4) and (4.5),

$$
\text { (4.16) } \begin{aligned}
\left(K_{\omega} g_{l}, g_{l}\right)_{\omega} & =2 \int_{\substack{\left|x-x_{0}\right| \leq\left|y-x_{0}\right| \\
\int_{0}}} \operatorname{Re}(M(x, y)) g_{l}(x) g_{l}(y) d x d y \\
& +2 \int_{\substack{\omega \\
\left|x-x_{0}\right| \leq\left|y-y_{0}\right|}} \int_{\omega} \operatorname{Re}(N(x, y)) g_{l}(x) g_{l}(y) d x d y
\end{aligned}
$$




$$
\begin{aligned}
& \leq-2 \sum_{i=1}^{N} d_{i} \int_{\tilde{\omega} \tilde{\tau}_{|\leq| y \mid}} \int_{\tilde{\omega} \mid}|x-y|^{\beta_{i}}|x|^{\gamma_{i}}|y|^{\delta_{i}} \tilde{g}_{l}(x) \tilde{g}_{l}(y) d x d y
\end{aligned}
$$

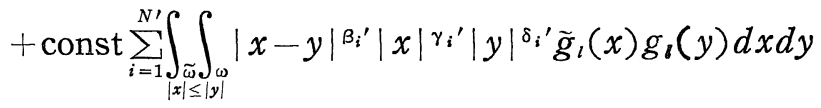

$$
\begin{aligned}
& =-2 \sum_{i=1}^{N} d_{i} l^{-\left(m+\beta_{i}+\gamma_{i}+\delta_{t}\right)} \\
& \times \int_{\substack{\omega \\
|x| \leq|y|}} \int_{\widetilde{\omega}}|x-y|^{\beta_{1}}|x|^{\gamma_{1}}|y|^{\delta_{i}} \tilde{g}_{1}(x) g_{1}(y) d x d y+\text { const } l^{-\left(\nu+\varepsilon^{\prime}\right)}
\end{aligned}
$$

Therefore, if we choose $l$ large enough, by (4.5) and (4.11) we have

$$
\begin{aligned}
\left(H_{\omega} g_{l}, g_{l}\right)_{\omega} & =\left(V_{\omega} g_{l}, g_{l}\right)_{\omega}+\left(K_{\omega} g_{l}, g_{l}\right)_{\omega} \\
& =-k_{0} l^{-\nu}+\mathrm{const} l^{-\left(\nu+\varepsilon^{\prime}\right)}<0 .
\end{aligned}
$$

By (4.7) and the inequality (4.17), there exists at least one negative discrete eigenvalue of $H_{\omega}$. Now it follows by (4.7) and by Lemma 4. 1 that $H$ has at least one negative discrete eigenvalue.

4. 4. (Proof of Theorem 4.1 continued) Now let the condition (4.3) -(4.10) be satisfied. We assume that there exist $p$ negative discrete eigenvalues of $H_{\omega}$. Call them $\left\{\lambda_{k}\right\}_{k=1, \ldots, p}$, and denote the associated eigenfunctions by $\left\{\varphi_{k}(x)\right\}_{k=1, \ldots, p}$ (these form an orthonormal system in $\left.L^{2}(\omega)\right)$. Setting

$$
v_{l}(x)=g_{l}(x)-\sum_{k=1}^{p} \beta_{l}^{(k)} \varphi_{k}(x), \quad \text { where } \quad \beta_{l}^{(k)}=\left(g_{l}, \varphi_{k}\right)_{\omega},
$$

we have

$$
\left(v_{l}, \varphi_{k}\right)_{\omega}=0 \quad(k=1, \cdots, p)
$$

By the orthonormality of $\left\{\varphi_{k}\right\}_{k=1, \cdots, p}$, we have

$$
\left(H_{\omega} v_{l}, v_{l}\right)_{\omega}=\left(H_{\omega} g_{l}, g_{l}\right)_{\omega}-\sum_{k=1}^{p} \lambda_{k}\left|\beta_{l}^{(k)}\right|^{2} .
$$

On the other hand, since $\lambda_{k} \neq 0$, and

we have

$$
\begin{aligned}
\left|\lambda_{k} \beta_{l}^{(k)}\right| & =\left|\left(g_{l}, H_{\omega} \varphi_{k}\right)_{\omega}\right|=\left|\left(H_{\omega} g_{l}, \varphi_{k}\right)_{\omega}\right| \\
& \leq\left|\left(V_{\omega} g_{l}, \varphi_{k}\right)_{\omega}\right|+\left|\left(K_{\omega} g_{l}, \varphi_{k}\right)_{\omega}\right| \quad(k=1, \cdots, p),
\end{aligned}
$$

$$
\left|\beta_{l}^{(k)}\right|^{2} \leq \mathrm{const}\left\|V_{\omega} g_{l}\right\|_{\omega}^{2}+\text { const }\left\|K_{\omega} g_{l}\right\|_{\omega}^{2},
$$


where the constant is independent of $l$. By (4.3), just as (4.15) was obtained,

$$
\left\|V_{\omega} g_{l}\right\|_{\omega}^{2} \leq \text { const } l^{-2 \nu} \text {. }
$$

Since by (4.8) we can make use of Fubini's theorem, it follows from (4.9), (4.10) and Lemma 4.1 that

$$
\begin{aligned}
\left\|K_{\omega} g_{l}\right\|_{\omega}^{2} & =\left(K_{\omega} K_{\omega} g_{l}, g_{l}\right)_{\omega} \\
& \leq \int_{\omega} \int_{\omega} K_{\omega}^{(2)}(x, y) g_{l}(x) g_{l}(y) d x d y<\mathrm{const} l^{-\left(\nu+\varepsilon^{\prime \prime}\right)} .
\end{aligned}
$$

Therefore, by (4.17) and (4.20)-(4.23),

$$
\left(H_{\omega} v_{l}, v_{l}\right)_{\omega}<0 \text { for large } l>0 \text {. }
$$

The relations (4.7), (4.19) and (4.24) show that at least $(p+1)$ negative discrete eigenvalues of $H_{\omega}$ exist. Then by mathematical induction, an infinite number of negative discrete eigenvalues of $H_{\omega}$ therefore exist. Thus, by (4.7) and Lemma 4.1, $H$ has an infinite number of negative discrete eigenvalues. (q. e. d.)

\section{$\S 5 . \quad$ Applications}

5. 1. Theorem 5.1. The Boltzmann operator $H$ which appears in the mono-atomic gas model for the neutron scattering problem in an infinite homogeneous medium is given by

$$
\begin{aligned}
(H f)(x) & =V(x) f(x)+\int_{0}^{\infty} K(x, y) f(y) d y \\
& =(V f)(x)+(K f)(x)(x \in(0, \infty)),
\end{aligned}
$$

where

$$
\begin{gathered}
V(x)=b\left[\left(x+\frac{1}{2 M x}\right) I(\sqrt{M x})+\frac{1}{\sqrt{\pi M}} e^{-M x^{2}}\right] \\
K(x, y)=-b \theta^{2}\left[e^{-1 / 2\left(y^{2}-x^{2}\right)}\{I(\theta x-\zeta y) \pm I(\theta x+\zeta y)\}\right. \\
\left.+e^{1 / 2\left(y^{2}-x^{2}\right)}\{I(\theta y-\zeta x) \mp I(\theta y+\zeta x)\}\right] \\
\quad \text { for } y \gtrless x, \\
I(x)=\frac{2}{\sqrt{\pi}} \int_{0}^{x} e^{-t 2} d t .
\end{gathered}
$$


Here $M$ and $b$ are positive constants $\theta=-\frac{M+1}{2 \sqrt{M}}$, and $\zeta=\frac{M-1}{2 \sqrt{M}}$. Then we have the following results:

(i) If the operator $H$ has the domain $D(H)=\{\varphi:(1+x) \varphi \in$ $\left.L^{2}(0, \infty)\right\}, H$ is a lower semi-bounded selfadjoint operator in $L^{2}(0, \infty)$.

(ii) If we put $\mu=V(+0)=\frac{2 b}{\sqrt{\pi M}}>0$,

(5.5) $\sigma_{e}(H)=[\mu, \infty)$.

(iii) $H$ has an infinite number of discrete eigenvalues in $(-\infty$, $\mu)^{14)}$

To show the above theorem we make use of the next lemma.

Lemma 5. 1. For $V(x)$ and $K(x, y)$ given by (5.2) and (5.3), we have

$$
\begin{aligned}
& V(x)=\mu+c x^{2}+W(x), \text { where } \quad c=\frac{2}{3} b \frac{\sqrt{M}}{\sqrt{\pi}} \text { and } \\
& |W(x)| \leq \text { const } x^{4} \quad(0<x<1),
\end{aligned}
$$

(5.7) $\quad V(x)$ is continuous in $(0, \infty)$, monotone increasing with

$$
\lim _{x \rightarrow \infty} \frac{V(x)}{x}=b,
$$

$$
|K(x, y)| \leq \mathrm{e}^{-\xi\left|x-y^{2}\right|} \text { for some } \xi>0,(0<x, y<+\infty),
$$

(5.9) $K(x, y)=K(y, x)$, and $K(x, y)$ is a real function, and

(5.10) $\quad K(x, y)=-d x+q(x, y)$,

where $d=\frac{4 \theta^{2}}{\sqrt{\pi}} b(\theta-\zeta)=\frac{(M+1)^{2}}{M^{3 / 2} \sqrt{\pi}} b$, and $|q(x, y)| \leq \operatorname{const} x\left(x^{2}+y^{2}\right)$ for $0<x \leq y<1$.

Proof. The assertions (5.6)-(5.9) are easily verified. The relation (5.10) obtains from the fact that for $y \geq x$,

$$
\begin{aligned}
K(x, y)= & \frac{-2 b \theta^{2}}{\sqrt{\pi}}\left[e^{-1 / 2\left(y^{2}-x^{2}\right)}\left\{\int_{0}^{\theta x-\zeta y} e^{-t^{2}} d t+\int_{0}^{\theta x+\zeta y} e^{-t^{2}} d t\right\}\right. \\
& \left.+e^{1 / 2\left(2 y-x^{2}\right)}\left\{\int_{0}^{\theta y-\zeta \varsigma^{-t^{2}}} d t-\int_{0}^{\theta y+\varsigma^{-t^{2}}} d t\right\}\right]
\end{aligned}
$$

14) Shizuta [10] has shown that $H \geq 0$, and that 0 is a simple eigenvalue of $H$. 


$$
\begin{aligned}
= & -\frac{2 b \theta^{2}}{\sqrt{\pi}}\left\{e^{-1 / 2\left(y^{2}-x^{2}\right)} \int_{\zeta y-\theta x}^{\theta x+\zeta y} e^{-t^{2}} d t-e^{1 / 2\left(y^{2}-x^{2}\right)} \int_{\theta y-\zeta x}^{\theta y+\zeta x} e^{t^{2}} d t\right\} \\
= & -\frac{4 b \theta^{2}}{\sqrt{\pi}} e^{-\eta y^{2}}\left\{e^{1 / 2 x^{2}} \int_{0}^{\theta x} e^{-t^{2}} \cosh (2 \zeta y t) d t\right. \\
& \left.-e^{-1 / 2 x^{2}} \int_{0}^{\zeta x} e^{-t^{2}} \cosh (2 \theta y t) d t\right\},
\end{aligned}
$$

where $\eta=\frac{M^{2}+1}{4 M}=\frac{1}{2}+\zeta^{2}=\theta^{2}-\frac{1}{2}$. (q. e. d.)

5. 2. Proof of Theorem 5.1. Set $\widetilde{V}(x)=V(x)-\mu$. Assumptions 1 -3 of (3.1) (with $\widetilde{H}$ for the $H$ of (3.1)) are satisfied by $\widetilde{H}=\widetilde{V}+$ $K$, and $\Omega=(0, \infty) \subset R^{1}$. By (5.7), $D(\widetilde{V})=\left\{\varphi ;(1+x) \varphi \in L^{2}(0, \infty)\right\}$. By (5.8), with $\alpha=1+\varepsilon(1>\varepsilon>0)$, for any $a>0$ there exists $c(a)>0$ such that $|\widetilde{V}(x)|^{\alpha} \leq a^{2}|\widetilde{V}(x)|^{2}+c(a)^{2}$, and

$$
\int_{0}^{\infty} \int_{0}^{\infty} \frac{|K(x, y)|^{2}}{(\widetilde{V}(y)+1)^{\alpha}} d x d y \leq \mathrm{const} \int_{0}^{\infty} \frac{d y}{(1+y)^{\alpha}} \int_{0}^{\infty} e^{-2 \xi\left|x^{2-y 2}\right|} d x<+\infty .
$$

Therefore, by Theorem 3.1, assertions (i) and (ii) follow directly. Now, by (5.6), for any $\eta>0$ there exists $\delta(0<\delta<1)$ such that $0 \leq$ $\widetilde{V}(x) \leq(c+\eta) x^{2}$ for $0<x<\delta$. Then (4.3)-(4.6) of Theorem 4.1 follow from Lemma $5.1\left(x_{0}=0, \omega=(0, \delta) \subset \Omega=(0, \infty) \subset R^{1}, \nu=2, \beta_{i}=0\right.$, $\left.\gamma_{i}=1, \delta_{i}=0, \varepsilon^{\prime}=2, m=1\right)$. In fact, putting $g(x)=1(x \in \omega)$ in (4.6), since for a sufficiently small $\eta>0$

$$
\begin{aligned}
(c+\eta) \int_{0}^{\delta} x^{2} d x-2 d \int_{0}^{\delta} d y \int_{0}^{y} x d x & =\frac{\delta^{3}}{3}(c+\eta-d) \\
& =\frac{-b \delta^{3}}{9 M^{3 / 2} \sqrt{\pi}}\left(M^{2}+6 M+3-\frac{3 \sqrt{\pi}}{b} M^{3 / 2} \eta\right)<0,
\end{aligned}
$$

(4.6) is satisfied. The relation (4.7) is easily verified by using Lemma 3.1 and Theorem 3.1. Finally, since from (5.10) follows $|K(x, y)| \leq$ const $x(1 \geq y \geq x>0)$, we have with $\delta>y \geq x>0$,

$$
\begin{aligned}
K_{\omega}^{(2)}(x, y) & =\int_{0}^{\delta}|K(x, z) K(z, y)| d z=\left\{\int_{0}^{x}+\int_{x}^{y}+\int_{y}^{\delta} d z\right\} \\
& \leq \operatorname{const}\left\{\int_{0}^{x} z^{2} d z+\int_{x}^{y} z x d z+\int_{y}^{\delta} x y d z\right\} \\
& \leq \operatorname{const}\left\{x^{3}+x\left(y^{2}+x^{2}\right)+x y+x y^{2}\right\} .
\end{aligned}
$$


For $\varepsilon^{\prime \prime}=1$ these results entail (4. 8)-(4.10). Therefore, since Theorem 4.1 implies that $\widetilde{H}$ has an infinite number of negative discrete eigenvalues, assertion (iii) is completely proved. (q. e. d.)

5.3. As another application of our method we consider the negative discrete eigenvalues of the Schrödinger operator in $L^{2}\left(R^{3}\right)$.

Definition 5. 1. Let $A$ and $B$ be symmetric in a Hilbert space $\mathfrak{S}$, and $D(A) \subset D(B)$. If $(A f, f) \geq(B f, f)$ for any $f \in D(A), A \geq B$.

The following fact is well-known.

Lemma 5. 2. If $A \geq B$ and both operators are selfadjoint in $\mathfrak{S}$, then we have $S(B:-\infty, 0) \geq S(A:-\infty, 0)$.

Proof. Let $M \subset D(A)$ be an $n$-dimensional subspace of $\mathfrak{S}$ such that for any $f \in M(f \neq 0),(A f, f)<0$ holds. Then for any $f \in M(f \neq$ $0),(B f, f)<0$. By Lemma 2.1, Lemma 5.2 follows. (q. e. d.)

We consider the Schrödinger operator which involving external magnetic field, which can be written in the form (5.11) below.

Theorem 5.2. Define $L_{0}$ by

$$
\left(L_{0} f\right)(x)=\sum_{k=1}^{3}\left(i \frac{\partial}{\partial x_{k}}+b_{k}(x)\right)^{2} f(x)+q_{0}(x) f(x),
$$

where

$$
\begin{aligned}
& b_{k}(x) \in \mathscr{B}^{1}\left(R^{3}\right) \text { and } q_{0}(x) \in L_{l o c}^{2}\left(R^{3}\right) \text { are real valued func- } \\
& \text { tions, }{ }^{15)} \text { and }
\end{aligned}
$$

Then:

$b_{k}(x), \frac{\partial}{\partial x_{k}} b_{k}(x)$ and $q_{0}(x)$ converge uniformly to zero as $|x| \rightarrow \infty$.

(i) If the domain of $L_{0}$ is $\mathscr{D}_{L^{2}}^{2}\left(R^{3}\right), L_{0}$ is a lower semi-bounded selfadjoint operator in $L^{2}\left(R^{3}\right) \cdot{ }^{16)}$

15) $f(x) \in \mathscr{B}^{1}\left(R^{3}\right)$ means that $f(x)$ has continuous derivatives of first order in $R^{3}$, and $\sup _{x \in R^{3}}|f(x)|+\sum_{k=1}^{3} \sup _{x \in R^{3}}\left|\frac{\partial f}{\partial x_{k}}(x)\right|<+\infty$.

16) $\mathscr{D}_{L^{2}}^{2}\left(R^{3}\right)$ is the completion of the space $C_{0}^{\infty}\left(R^{3}\right)$ with the norm

$$
\left\|f_{\|}\right\|_{2, L^{2}\left(R^{3}\right)}=\left\{\|f\|_{L^{2}\left(R^{3}\right)}^{2}+\sum_{k=1}^{3}\left\|\frac{\partial f}{\partial x_{k}}\right\|_{L^{2}\left(R^{3}\right)}^{2}+\sum_{k, l=1}^{3}\left\|\frac{\partial^{2} f}{\partial x_{k} \partial x_{l}}\right\|_{L^{2}\left(R^{3}\right)}^{2},\right.
$$

where $C_{0}^{\infty}\left(R^{3}\right)$ is the space of all $C^{\infty}$ functions with compact support. 
(ii) $\sigma_{e}\left(L_{0}\right)=[0, \infty)$.

(iii) (a) If there exist $\alpha>0$ and $R_{0}>0$ such that for all $|x| \geq$ $R_{0}$.

$$
\frac{1}{\alpha} \sum_{k=1}^{3} b_{k}^{2}(x)+\frac{1}{1+\alpha} q_{0}(x) \leq-\frac{c}{|x|^{2}}
$$

(where $c>\inf _{g \in W(\omega)}\left\{4 \pi \int_{\omega}|x|^{2}|g(x)|^{2} d x\left(\int_{\omega} \int_{\omega} \frac{g(x) g(y)}{|x-y|} d x d y\right)^{-1}\right\}, \quad \omega=\{x \in$ $\left.R^{3}:|x|<1\right\}$ and $W(\omega)=\left\{g \in L^{2}(\omega):\|g\|_{\omega}=1, g(x) \geq 0\right.$ for a. e. $x \in$ $\omega\}$ ), then $L_{0}$ has an infinite number of negative discrete eigenvalues.

(b) If there exist $\alpha^{\prime}\left(1>\alpha^{\prime}>0\right)$ and $q_{2}(x) \in L^{2}\left(R^{3}\right)$ such that $\frac{1}{1-\alpha^{\prime}} q_{0}(x)-\frac{1}{\alpha^{\prime}} \sum_{k=1}^{3} b_{k}^{2}(x) \geq q_{2}(x)$, and if $\frac{\hat{q}_{2}(x-y)}{|x||y|}$ is either a kernel of a completely continuous selfadjoint integral operator in $L^{2}\left(R^{3}\right)$ or is a bounded selfadjoint integral operator with norm smaller than $4 \pi^{2}$, then $L_{0}$ has at most a finite number of negative discrete eigenvalues. ${ }^{17)}$

Proof of Theorem 5.2. Assertion (i) is clear by results of Ikebe-Kato [5] or Jörgens [6]. Assertion (ii) is also clear by a result of Jörgens [6]. We shall show that assertion (iii) (a) holds.

For any $f \in \mathscr{D}_{L^{2}}^{2}\left(R^{3}\right)$,

$$
\begin{aligned}
\left(L_{0} f, f\right)_{L^{2}\left(R^{3}\right)}= & \sum_{k=1}^{3}\left(\left(i \frac{\partial f}{\partial x_{k}}+b_{k} f\right),\left(i \frac{\partial f}{\partial x_{k}}+b_{k} f\right)\right)_{L^{2}\left(R^{3}\right)} \\
& +\left(q_{0} f, f\right)_{L^{2}\left(R^{3}\right)} \leq(1+\alpha)\left\{(-\Delta f, f)_{L^{2}\left(R^{3}\right)}\right. \\
& \left.+\left(\left(\frac{1}{\alpha} \sum_{k=1}^{3} b_{k}^{2}+\frac{1}{1+\alpha} q_{0}\right) f, f\right)_{L^{2}\left(R^{3}\right)}\right\} .
\end{aligned}
$$

Let

$$
q_{1}(x)= \begin{cases}\frac{1}{\alpha} \sum_{k=1}^{3} b_{k}^{2}(x)+\frac{1}{1+\alpha} q_{0}(x) & :|x|<R_{0} \\ -\frac{c}{|x|^{2}} & :|x| \geq R_{0}\end{cases}
$$

and

$$
L_{1}=-\Delta+q_{1}(x)
$$

17) $q_{2}(\xi)$ denotes the Fourier transform of $q_{2}(x)$, which is given by (5.17). 
so that $(1+\alpha) L_{1}>L_{0}$. With $D\left(L_{1}\right)=\mathscr{D}_{L^{2}}^{2}\left(R^{3}\right), L_{1}$ is selfadjoint in $L^{2}\left(R^{3}\right)$. On the other hand, the Fourier transform

$$
(F f)(\xi) \equiv \hat{f}(\xi) \equiv \text { l. i. m. } \int_{R \rightarrow \infty} f(x) e^{-2 \pi i x \cdot \xi} d x
$$

(where $f(x) \in L^{2}\left(R^{3}\right)$ and $\left.x \cdot \xi=\sum_{i=1}^{3} x_{i} \xi_{i}\right)$ is a unitary transformation on $L^{2}\left(R^{3}\right)$. And for any $f \in \mathscr{D}_{L^{2}}^{2}\left(R^{3}\right), \hat{f}(\xi) \in L^{1}\left(R^{3}\right)$. Indeed, $\mathscr{D}_{L^{2}}^{2}\left(R^{3}\right)=$ $\left\{f(x) \in L^{2}\left(R^{3}\right):\left(1+|\xi|^{2}\right) \hat{f}(\xi) \in L^{2}\left(R^{3}\right)\right\}$, so that

$$
\int_{R^{3}}|\hat{f}(\xi)| d \xi \leq \sqrt{\int_{R^{3}} \frac{d \xi}{\left(1+|\xi|^{2}\right)^{2}}} \sqrt{\int_{R^{3}}\left(1+|\xi|^{2}\right)^{2}|\hat{f}(\xi)|^{2} d \xi}<+\infty .
$$

By the definition of $q_{1}(x)$, we have $q_{1}(x) \in L^{2}\left(R^{3}\right)$. Let $\left(H_{1} \hat{f}\right)(\xi)=$ $\left(F L_{1} F^{-1} \hat{f}\right)(\xi)$. Then for $f \in \mathscr{D}_{L^{2}}^{2}\left(R^{3}\right)$,

$$
\left(H_{1} \hat{f}\right)(\xi)=4 \pi^{2}|\xi|^{2} \cdot \hat{f}(\xi)+\int \widehat{q}_{R^{3}}(\xi-\eta) \hat{f}(\eta) d \eta .
$$

It follows easily that Assumptions 1-3 of (3.1) (with $H_{1}$ as $H$ ) are satisfied for $H_{1}$ and $\Omega=R^{3}$, and that $H_{1}$ is a selfadjoint operator with the domain $D\left(H_{1}\right)=\left\{\hat{f} \in L^{2}\left(R^{3}\right) ;\left(1+|\xi|^{2}\right) \hat{f}(\xi) \in L^{2}\left(R^{3}\right)\right\}$. We have

$$
\begin{aligned}
& \lim _{R \rightarrow \infty} \int_{R_{0} \leq|x| \leq R} \frac{e^{-2 \pi i x \cdot \xi}}{|x|^{2}} d x=\lim _{R \rightarrow \infty} 2 \pi \int_{R_{0}}^{R} \frac{r^{2} d r}{r^{2}} \int_{0}^{\pi} e^{-2 \pi i r|\xi| \cos \theta} \sin \theta d \theta \\
& \quad=\lim _{R \rightarrow \infty} 2 \pi \int_{R_{0}}^{R} d r \int_{-1}^{1} e^{-2 \pi i r|\xi| t} d t=\lim _{R \rightarrow \infty} \int_{R_{0}}^{R} \frac{2 \sin (2 \pi r|\xi|)}{|\xi| r} d r \\
& \quad=\lim _{R \rightarrow \infty} \frac{2}{|\xi|} \int_{2 \pi R_{0}|\xi|}^{2 \pi R|\xi|} \frac{\sin r}{r} d r .
\end{aligned}
$$

Since $\lim _{R \rightarrow \infty} \int_{0}^{R} \frac{\sin r}{r} d r=\frac{\pi}{2}$,

$$
\left|\lim _{R \rightarrow \infty} \int_{R_{0} \leq|x| \leq R} \frac{e^{-2 \pi i x \cdot \xi}}{|x|^{2}} d x-\frac{\pi}{|\xi|}\right| \leq \frac{1}{|\xi|} \int_{0}^{2 \pi R_{0}|\xi|} \frac{|\sin r|}{r} d r \leq \text { const for }|\xi| \leq 2 .
$$

Since $q_{1}(x) \in L^{2}\left(R^{3}\right), q_{1}(x) \in L_{l o c}^{1}\left(R^{3}\right)$. And for $|\xi-\eta| \leq 2$,

$$
\hat{q}_{1}(\xi-\eta)=-\frac{c \pi}{|\xi-\eta|}+p(\xi-\eta)
$$


(where $|p(\xi-\eta)| \leq$ const).

It is clear that $H_{1}$ satisfies conditions (4.3)-(4.6) of Theorem 4.1 with $x_{0}=0, \nu=2, \beta_{i}=-1, \gamma_{i}=\delta_{i}=0, m=3, \varepsilon^{\prime}=1$, and $\omega=\left\{x \in R^{3}:|x|\right.$ $<1$. As for (4.7), in view of the inequality

$$
\begin{aligned}
\int_{R^{3}} \int_{R 3} \frac{\left|\hat{q}_{1}(\xi-\eta)\right|^{2} d \xi d \eta}{\left(1+|\xi|^{2}\right)\left(1+|\eta|^{2}\right)} & =2 \int_{R^{3}} \int_{R 3} \frac{\left|\hat{q}_{1}(\xi-\eta)\right|^{2} d \xi d \eta}{\left(1+|\xi|^{2}\right)\left(1+|\eta|^{2}\right)} \\
& \leq 2 \int_{R 3} \frac{d \eta}{\left(1+|\eta|^{2}\right)^{2}} \int_{R 3}\left|\hat{q}_{1}(\xi-\eta)\right|^{2} d \xi<+\infty
\end{aligned}
$$

$\sigma_{e}\left(H_{1}\right)=[0, \infty)$ and $\sigma_{e}\left(H_{1, \omega}\right)=\left[0,4 \pi^{2}\right]$ by Theorem 2.1 and Lemma 3.1. Conditions (4.8)-(4.10) are satisfied for $\varepsilon^{\prime \prime}=1$, since by (5.20), for $\xi, \eta \in \omega$,

$$
\int_{|\zeta| \leq 1}\left|\hat{q}_{1}(\xi-\zeta) \hat{q}_{1}(\zeta-\eta)\right| d \zeta \leq \text { const } \int_{|\zeta| \leq 1} \frac{d \zeta}{|\xi-\zeta||\zeta-\eta|} \leq \text { const. }
$$

Thus $H_{1}$ has an infinite number of negative discrete eigenvalues, and hence the same is true for $L_{1}$. In view of Lemma 5.2 and (ii), (iii) (a) follows.

Now we shall show that assertion (iii) (b) holds. With

$$
L_{2}=-\Delta+q_{2}(x),
$$

the relation $L_{0} \geq\left(1-\alpha^{\prime}\right) L_{2}$ follows in a way similar to (5.14). With $D\left(L_{2}\right)=\mathscr{D}_{L^{2}}^{2}\left(R^{3}\right), L_{2}$ is selfadjoint in $L^{2}\left(R^{3}\right)$. Therefore, if we define $\left(H_{2} \hat{f}\right)(\xi)=\left(F L_{2} F^{-1} \hat{f}\right)(\xi)$ for $f \in \mathscr{D}_{L^{2}}^{2}\left(R^{3}\right), H_{2}$ is selfadjoint in $L^{2}\left(R^{3}\right)$, and

$$
\left(H_{2} \hat{f}\right)(\xi)=4 \pi^{2}|\xi|^{2} \hat{f}(\xi)+\int_{R 3} \hat{q}_{2}(\xi-\eta) \hat{f}(\eta) d \eta
$$

holds for $f \in \mathscr{D}_{L^{2}}^{2}\left(R^{3}\right)$. Thus by Theorem 3.2 or Corollary 2.1 , there exist at most a finite number of negative discrete eigenvalues of $H_{2}$ and, hence, of $L_{2}$. Thus in view of (ii) and Lemma 5.2, we have (iii) (b). (q. e. d.)

Remark 5.1. If there exist $\alpha^{\prime}\left(1>\alpha^{\prime}>0\right)$ and $R_{0}^{\prime}>0$ such that for all $|x| \geq R_{0}^{\prime}$, 


$$
\frac{1}{1-\alpha^{\prime}} q_{0}(x)-\frac{1}{\alpha^{\prime}} \sum_{k=1}^{3} b_{k}^{2}(x) \geq-\frac{\text { const }}{|x|^{2+\varepsilon}} \quad(\varepsilon>0)
$$

then the conditions of Theorem 5.2 (iii) (b) are satisfied. In fact, set

$$
q_{2}(x)= \begin{cases}\frac{1}{1-\alpha^{\prime}} q_{0}(x)-\frac{1}{\alpha^{\prime}} \sum_{k=1}^{3} b_{k}^{2}(x) & :|x|<R_{0}^{\prime} \\ -\frac{\text { const }}{|x|^{2+\varepsilon}} & :|x| \geq R_{0}^{\prime} .\end{cases}
$$

Then $q_{2}(x)$ satisfies the conditions of Theorem 5.2 (iii)(b). Indeed. we have similarly to (5.19).

$$
\begin{aligned}
\left|\lim _{R \rightarrow \infty} \int_{R_{0^{\prime}} \leq|x|<R} \frac{e^{-2 \pi i x \cdot \xi}}{|x|^{2+\varepsilon}} d x\right| & =\mid \frac{2(2 \pi)^{\varepsilon}}{|\xi|^{1-\varepsilon}} \lim _{R \rightarrow \infty}^{2 \pi R|\xi|} \int_{2 \pi R_{0}|\xi|}^{\frac{\sin r}{r^{1+\varepsilon}} d r \mid} \\
& \leq \frac{\text { const }}{|\xi|^{1-\varepsilon}}\left\{\int_{1}^{\infty} \frac{d r}{r^{1+\varepsilon}}+\int_{0}^{1} \frac{\sin r}{r^{1+\varepsilon}} d r\right\} \leq \frac{\text { const }}{|\xi|^{1-\varepsilon}} .
\end{aligned}
$$

Since $q_{2}(x) \in L^{2}\left(R^{3}\right) \subset L_{\mathrm{loc}}^{1}\left(R^{3}\right)$, for $|\xi-\eta| \leqq 1$.

$$
\left|\hat{q}_{2}(\xi-\eta)\right| \leq \frac{\text { const }}{|\xi-\eta|^{1-\varepsilon}} .
$$

Setting $G_{0}(\xi, \eta)=\frac{q_{2}(\xi-\eta)}{|\xi||\eta|}$ gives

$$
\int_{R^{3}} \int_{R^{3}}\left|G_{0}(\xi, \eta)\right|^{2} d \xi d \eta=\int_{|\xi| \leq 1} \int_{|\eta| \leq 1}+2 \int_{|\xi| \geq 1} \int_{|\eta| \leq 1}+\int_{|\xi| \geq 1} \int_{|\eta| \geq 1}=I_{1}+I_{2}+I_{3} .
$$

By (5.24) and the fact that $q_{2}(\xi) \in L^{2}\left(R^{3}\right)$,

$$
\begin{aligned}
& I_{1} \leq \text { const } \int_{|\xi| \leq 1} \frac{d \xi}{|\xi|^{2}} \int_{|\eta| \leq 1} \frac{d_{\eta}}{|\eta|^{2}|\xi-\eta|^{2-2 \varepsilon}} \leq \text { const } \int_{|\xi| \leq 1} \frac{d \xi}{|\xi|^{2}|\xi|^{1-2 \varepsilon}}<+\infty, \\
& I_{2} \leq 2 \int_{|\eta| \leq 1} \frac{d \eta}{|\eta|^{2}} \int_{R^{3}}\left|\hat{q}_{2}(\xi-\eta)\right|^{2} d \xi<+\infty, \text { and } \\
& I_{3} \leq 2 \iint_{|\xi| \geq|\eta| \geq 1} \frac{\left|\hat{q}_{2}(\xi-\eta)\right|^{2}}{|\eta|^{4}} d \xi d \eta \leq 2 \int_{|\eta| \geq 1} \frac{d \eta}{|\eta|^{4}} \int_{R 3}\left|\hat{q}_{2}(\xi-\eta)\right|^{2} d \xi<+\infty .
\end{aligned}
$$

Thus $G_{0}$ is a completely continuous operator in $L^{2}\left(R^{3}\right)$ with kernel $G_{0}(x, y)$ of Hilbert-Schmidt type, and the assertion is proved. (q. e. d.)

5. 4. Finally we consider the so-called Friedrichs model. 
Theorem 5.3. Let

$$
(H f)(x)=x \cdot f(x)+\int_{0}^{\infty} K(x, y) f(y) d y \quad(0<x<\infty),
$$

where

$$
\begin{cases}K(x, y)=\overline{K(y, x)} & \\ |K(x, y)| \leq \mathrm{const}(1+x+y)^{-\theta} & (\theta>1 / 2) \\ |K(x, y)| \leq \mathrm{const}|x|^{\mu} & (\mu>0) \text { for } 0<x, y<1 .\end{cases}
$$

Then:

(i) With $D(H)=\left\{f \in L^{2}(0, \infty) ; x f(x) \in L^{2}(0, \infty)\right\}, H$ defines a lower semi-bounded selfadjoint operator in $L^{2}(0, \infty)$.

(ii) $\sigma_{e}(H)=[0, \infty)$.

(iii) There exist at most a finite number of negative discrete eigenvalues of $H$.

Proof. In this case $\Omega=(0, \infty) \subset R^{1}$. For any $f \in D(H), K f \in$ $L^{2}(0, \infty)$ because, by $(5.25)$,

$$
|(K f)(x)| \leqq\left.\frac{\text { const }}{(1+x)^{\theta}} \sqrt{\int_{0}^{\infty} \frac{d y}{(1+y)^{2}}} \sqrt{\int_{0}^{\infty}(1+y)^{2} \mid f(y)}\right|^{2} d y \leqq \frac{\text { const }}{(1+x)^{\theta}} .
$$

Thus, if $D(H)$ is taken to be the domain of $K, K$ is a symmetric operator in $L^{2}(0, \infty)$. Then Assumptions 1-3 of (3.1) are satisfied by $H$. If $\alpha$ satisfies $1<\alpha<2$, then for any $a>0$, we can choose a $c(a)>0$ so that $x^{\alpha} \leqq a^{2} x^{2}+c(a)^{2}$ for all $x \in(0, \infty)$.

Also by (5.25),

$$
\int_{0}^{\infty} \int_{0}^{\infty} \frac{|K(x, y)|^{2}}{(1+y)^{\alpha}} d x d y \leq \mathrm{const} \int_{0}^{\infty} \frac{d x}{(1+x)^{2 \theta}} \int_{0}^{\infty} \frac{d y}{(1+y)^{\alpha}}<+\infty .
$$

Now Theorem 3.1 and Lemma 3.1 imply the truth of assertions (i) and (ii). Setting $G_{0}(x, y)=\frac{K(x, y)}{\sqrt{x} \sqrt{y}}$, we have

$$
\begin{aligned}
\int_{0}^{\infty} \int_{0}^{\infty}\left|G_{0}(x, y)\right|^{2} d x d y= & \int_{0}^{\infty} \int_{0}^{\infty} \frac{|K(x, y)|^{2}}{x y} d x d y \\
= & \int_{0}^{1} \int_{0}^{1} \frac{|K(x, y)|^{2}}{x y} d x d y+2 \int_{0}^{1} d x \int_{1}^{\infty} \frac{|K(x, y)|^{2}}{x y} d y \\
& +\int_{1}^{\infty} \int_{1}^{\infty} \frac{|K(x, y)|^{2}}{x y} d x d y=I_{1}+I_{2}+I_{3} .
\end{aligned}
$$


But we also have $|K(x, y)| \leqq$ const $|y|^{\mu}$ for $0<x, y<1$ by the first and third relations in (5.25). Hence, the following estimates hold:

$$
\begin{aligned}
& I_{1} \leq \mathrm{const} \int_{0}^{1} \frac{d x}{x^{1-\mu}} \int_{0}^{1} \frac{d y}{y^{1-\mu}}<+\infty \\
& I_{2} \leq \mathrm{const} \int_{0}^{1} \frac{d x}{x^{1-\mu}} \int_{1}^{\infty} \frac{|K(x, y)|}{y} d y \leq \mathrm{const} \int_{0}^{1} \frac{d x}{x^{1-\mu}} \int_{1}^{\infty} \frac{d y}{y(1+y)^{\theta}}<+\infty,
\end{aligned}
$$

and

$$
\begin{aligned}
I_{3} \leq \mathrm{const} \int_{1}^{\infty} \int_{1}^{\infty} & \frac{d x d y}{x y(1+x+y)^{2 \theta}} \\
& \leq \mathrm{const} \int_{1}^{\infty} \frac{d x}{x(1+x)^{\theta}} \int_{1}^{\infty} \frac{d y}{y(1+y)^{\theta}}<+\infty .
\end{aligned}
$$

Thus $G_{0}$ is completely continuous. Corollary 2.1 and Theorem 3.2 now lead to the required result (iii). (q. e. d.)

Remark 5.2. The spectrum of the above operator has been investigated by Faddeev [2]. He introduced a Banach space consisting of Hölder-continuous functions, and used it to show the above results. But in studying the discrete spectrum, we may make use of the above method to by-pass the introduction of such a Banach space.

Theorem 5.4. Let

$$
(H f)(x)=|x|^{2} f(x)+\int_{R^{n}} K(x, y) f(y) d y \quad\left(x \in R^{n}, n \geq 3\right),
$$

where

$$
\left\{\begin{array}{l}
K(x, y)=\overline{K(y, x)} \\
|K(x, y)| \leq \frac{\text { const }}{(1+|x-y|)^{n+\varepsilon}} \quad(\varepsilon>0) .
\end{array}\right.
$$

Then;

(i) With domain $D(H)=\left\{f(x) ;\left(1+|x|^{2}\right) f(x) \in L^{2}\left(R^{n}\right)\right\}, \quad H$ defines a selfadjoint operator in $L^{2}\left(R^{n}\right)$;

(ii) $\sigma_{e}(H)=[0, \infty)$;

(iii) There exist at most a finite number of negative discrete eigenvalues of $H$.

Proof. Here, $\Omega=R^{n}$. Assertion (i) is clear because (5. 27) implies that $K$ is a bounded selfadjoint operator. And Assumptions 1-3 of (3.1) are also satisfied. We set 


$$
\left(G_{0} f\right)(x)=\int_{R^{n}} \frac{K(x, y)}{|x||y|} f(y) d y=\sum_{i=1}^{4}\left(G_{0, R}^{(i)} f\right)(x),
$$

where $\left\{G_{0, R}^{(i)}\right\}_{i=1, \ldots, 4}$ are integral operators with kernels

$$
\begin{aligned}
& G_{0, R}^{(1)}(x, y)= \begin{cases}G_{0}(x, y): & |x|,|y| \leq R \\
0 & : \text { otherwise }\end{cases} \\
& G_{0, R}^{(2)}(x, y)= \begin{cases}G_{0}(x, y): & |x|>R \geq|y| \\
0 & : \text { otherwise, }\end{cases} \\
& G_{0, R}^{(3)}(x, y)= \begin{cases}G_{0}(x, y): & |y|>R \geq|x| \\
0 & : \text { otherwise }\end{cases} \\
& G_{0, R}^{(4)}(x, y)= \begin{cases}G_{0}(x, y):|x|,|y|>R \\
0: \text { otherwise. }\end{cases}
\end{aligned}
$$

Then

$$
\iint_{R^{n}}\left|G_{0, R}^{(1)}(x, y)\right|^{2} d x d y \leq \mathrm{const} \int_{|x| \leq R} \frac{d x}{|x|^{2}} \int_{|y| \leq R} \frac{d y}{|y|^{2}}<+\infty .
$$

And for any $f \in L^{2}\left(R^{n}\right)$,

$$
\begin{aligned}
& \int_{R^{n}} d x\left|\int_{R^{n}} G_{0, R}^{(2)}(x, y) f(y) d y\right|^{2} \\
& \leq \frac{\text { const }}{R^{2}} \int_{R^{n}} d x\left(\int_{R^{n}} \frac{d y}{|y|^{2}(1+|x-y|)^{n+\varepsilon}}\right)\left(\int_{R^{n}} \frac{|f(y)|^{2} d y}{(1+|x-y|)^{n+\varepsilon}}\right) \\
& \leq \frac{\text { const }}{R^{2}} \int_{R^{n}} d x\left(\int_{R^{n}} \frac{|f(y)|^{2} d y}{(1+|x-y|)^{n+\varepsilon}}\right)\left(\int_{|y| \leq 1} \frac{d y}{|y|^{2}}+\int_{R^{n}} \frac{d y}{(1+|x-y|)^{n+\varepsilon}}\right) \\
& \leq \frac{\text { const }}{R^{2}} \int_{R^{n}}|f(y)|^{2} d y \int_{R^{n}} \frac{d x}{(1+|x-y|)^{n+\varepsilon}} \leq \frac{\text { const }}{R^{2}}\|f\|_{L^{2}\left(R^{n}\right)}^{2} .
\end{aligned}
$$

In a similar fashion we have

$$
\left\|G_{0, R}^{(3)} f\right\|_{L^{2}\left(R^{n}\right)} \leq \frac{\text { const }}{R}\|f\|_{L^{2}\left(R^{n}\right)}
$$

and

$$
\left\|G_{0, R}^{(4)} f\right\|_{L^{2}\left(R^{n}\right)} \leq \frac{\text { const }}{R^{2}}\|f\|_{L^{2}\left(R^{n}\right)} .
$$

Let $M \subset L^{2}\left(R^{n}\right)$ satisfy $\|f\|_{L^{2}\left(R^{n}\right)} \leq C$ for any $f \in M$. For any $\eta>0$ there exists an $R$ large enough to satisfy $\left\|\left(G_{0, R}^{(2)}+G_{0, R}^{(3)}+G_{0, R}^{(4)}\right) f\right\|_{L^{2}\left(R^{n}\right)}<\eta$. But, because $G_{0, R}^{(1)}$ is completely continuous, there exists a sequence of elements $\left\{f_{k}\right\}_{k=1,2 \ldots} \subset M$ such that $\lim _{k, m \rightarrow \infty}\left\|G_{0, R}^{(1)} f_{k}-G_{0, R}^{(1)} f_{m}\right\|_{L^{2}\left(R^{n}\right)}=0$. Since 
$\varlimsup_{k, m \rightarrow \infty}\left\|G_{0} f_{k}-G_{0} f_{m}\right\|_{L^{2}\left(K^{n}\right)} \leq \eta$, application of the diagonal method gives a subsequence $\left\{f_{k p}\right\} \subset\left\{f_{k}\right\}$ such that $\left\{G_{0} f_{k_{p}}\right\}$ is a Cauchy sequence. Corsequently, $G_{0}$ is a completely continuous operator in $L^{2}\left(R^{n}\right)$, and so by Corollary 2.1 and Theorem 3.2 statements (ii) and (iii) hold. (q. e. d.)

Remark 5.3. Ushijima [13] has investigated the spectra of the operators of the type given in Theorem 5.4. But for the discrete spectrum he has only shown that $\sigma_{e}(H) \cap(-\infty, 0)=Q$.

In conclusion, the writer wishes to express his sincere gratitude to Professor T. Ikebe for his kind advice.

\section{REFERENCES}

[1] Birman, M. ̌., On the spectrum of singular boundary-value problems (Russian), Mat. Sb. 55 (1961), 125-174.

[2] Faddeev, L.D., On the Friedrichs model in the perturbation theory of continuous spectra (Russian), Trudy Mat. Inst. Steklov. 73 (1964), 292-313.

[3] Friedrichs, K.O., Über die Spektralzerlegung eines Integraloperators, Math. Ann. 115 (1938), 249-272.

[4] . On the perturbation of continuous spectra, Comm. Pure Appl. Math. 1 (1948), 361-406.

[5] Ikebe, T. and T. Kato, Uniqueness of the selfadjoint extension of singular elliptic differential operators, Arch. Rational Mech. Anal. 9 (1962), 77-92.

[6] Jörgens, K., Über das wesentliche Spektrum elliptischer Differentialoperatoren vom Schrödinger-Typ, to apper.

[7] Kato, T., Fundamental properties of Hamiltonian operators of Schrödinger type, Trans. Amer. Math. Soc. 70 (1951), 195-211.

[8] Kuščer, I. and N. Corngold, Discrete relaxation times in neutron thermalization, Phys. Rev. 139 (1965), 981-990.

[9] Mizohata, S. and K. Mochizuki, On the point spectrum of the Schrödinger operator, Proc. Japan Acad. 39 (1963), 661-666.

[10] Shizuta, Y., On fundamental equations of spatially independent problems in neutron thermalization theory, Prog. Theort. Phys. 32 (1964), 489-511.

[11] Uchiyama, J., On the discrete eigenvalues of the many-particle system, Publ. RIMS, Kyoto Univ. Ser. A 2 (1966), 117-132.

[12] Ukai, S., On the spectrum of the space-independent Boltzmann operator, J. Nuclear Energy 19 (1965), 833-848.

[13] Ushijima, T., On the spectrum of some Hamiltonian operator, Sci. Papers College Gen. Ed. Univ. Tokyo 16 (1966), 127-133.

[14] Žislin, G.M., A study of the spectrum of the Schrödinger operator for a system of several particles (Russian), Trudy Moskov. Mat. Obšč. 9 (1960), 82-120. 
